\title{
Reconciling Lambek's Restriction, Cut-Elimination, and Substitution in the Presence of Exponential Modalities
}

\author{
Max Kanovich \\ University College London and \\ National Research University Higher School of Economics (Moscow) \\ Stepan Kuznetsov \\ Steklov Mathematical Institute (Moscow) and \\ National Research University Higher School of Economics (Moscow) \\ Andre Scedrov \\ University of Pennsylvania and \\ National Research University Higher School of Economics (Moscow)
}

\begin{abstract}
The Lambek calculus can be considered as a version of non-commutative intuitionistic linear logic. One of the interesting features of the Lambek calculus is the so-called "Lambek's restriction," that is, the antecedent of any provable sequent should be non-empty. In this paper we discuss ways of extending the Lambek calculus with the linear logic exponential modality while keeping Lambek's restriction. Interestingly enough, we show that for any system equipped with a reasonable exponential modality the following holds: if the system enjoys cut elimination and substitution to the full extent, then the system necessarily violates Lambek's restriction. Nevertheless, we show that two of the three conditions can be implemented. Namely, we design a system with Lambek's restriction and cut elimination and another system with Lambek's restriction and substitution. For both calculi we prove that they are undecidable, even if we take only one of the two divisions provided by the Lambek calculus. The system with cut elimination and substitution and without Lambek's restriction is folklore and known to be undecidable.
\end{abstract}

Keywords: Lambek calculus, linear logic, exponential modalities, Lambek's restriction, cut elimination, substitution, undecidability 


\section{Introduction}

\subsection{The Lambek Calculus}

The Lambek calculus was introduced by J. Lambek in [1] for mathematical description of natural language syntax by means of so-called Lambek categorial (type-logical) grammars (see, for example, [2, 3, 4] ). In Lambek grammars, syntactic categories are represented by logical formulae involving three connectives: the product (corresponds to concatenation of words) and two divisions (left and right), and syntactic correctness of natural language expressions corresponds to derivability in the Lambek calculus.

For simplicity, in this paper we discuss only the product-free fragment of the Lambek calculus. First we consider not the Lambek calculus L [1], but its variant $\mathbf{L}^{*}[5]$. The difference between $\mathbf{L}$ and $\mathbf{L}^{*}$ is explained in the end of this introductory section (see "Lambek's Restriction").

$\mathbf{L}^{*}$ is a substructural logic, and here we formulate it as a Gentzen-style sequent calculus. Formulae of $\mathbf{L}^{*}$ are called types and are built from variables, or primitive types $\left(p, q, r, p_{1}, p_{2}, \ldots\right)$ using two binary connectives: \left } division) and / (right division). Types are denoted by capital Latin letters; finite (possibly empty) linearly ordered sequences of types by capital Greek ones. $\Lambda$ stands for the empty sequence. The Lambek calculus derives objects called sequents of the form $\Pi \rightarrow A$, where the antecedent $\Pi$ is a linearly ordered sequence of types and the succedent $A$ is a type.

The axioms of $\mathbf{L}^{*}$ are all sequents $A \rightarrow A$, where $A$ is a type, and the rules of inference are as follows:

$$
\begin{array}{ll}
\frac{A, \Pi \rightarrow B}{\Pi \rightarrow A \backslash B}(\rightarrow \backslash) & \frac{\Pi \rightarrow A}{\Delta_{1}, \Pi, A \backslash B, \Delta_{1}, B, \Delta_{2} \rightarrow C}(\backslash \rightarrow) \\
\frac{\Pi, A \rightarrow B}{\Pi \rightarrow B / A}(\rightarrow /) & \frac{\Pi \rightarrow A \quad \Delta_{1}, B, \Delta_{2} \rightarrow C}{\Delta_{1}, B / A, \Pi, \Delta_{2} \rightarrow C}(/ \rightarrow)
\end{array}
$$

For $\mathbf{L}^{*}$ and other calculi introduced later in this paper, we do not include cut as an official rule of the system. However, the cut rule of the following non-commutative form

$$
\frac{\Pi \rightarrow A \quad \Delta_{1}, A, \Delta_{2} \rightarrow B}{\Delta_{1}, \Pi, \Delta_{2} \rightarrow B}(\text { cut })
$$

is admissible in $\mathbf{L}^{*}[5]$.

By $\mathbf{L}_{/}^{*}$ (resp., $\mathbf{L}_{\backslash}^{*}$ ) we denote the fragment of $\mathbf{L}^{*}$ with only the right (resp., left) division connective. Due to the subformula property, these fragments are obtained from the full calculus simply by restricting the set of rules. 


\subsection{The Exponential Modality}

We see that $\mathbf{L}^{*}$ lacks structural rules (except for the implicit rule of associativity).

L* can be conservatively embedded [6, 7] into a non-commutative, intuitionistic or cyclic, variant of Girard's [8] linear logic. In the spirit of linear logic connectives, the Lambek calculus can be extended with the exponential unary connective that enables structural rules (weakening, contraction, and commutativity) in a controlled way.

We'll denote this extended calculus by EL*. Types of EL* are built from variables using two binary connectives ( $\backslash$ and /) and a unary one, !, called the exponential, or, colloqually, "bang." If $\Gamma=A_{1}, \ldots, A_{k}$, then by ! $\Gamma$ we denote the sequence $! A_{1}, \ldots, ! A_{k}$. EL $\mathbf{L}^{*}$ is obtained from $\mathbf{L}^{*}$ by adding the following rules:

$$
\begin{array}{cc}
\frac{\Delta_{1}, A, \Delta_{2} \rightarrow B}{\Delta_{1}, ! A, \Delta_{2} \rightarrow B}(! \rightarrow) & \frac{! \Gamma \rightarrow A}{! \Gamma \rightarrow ! A}(\rightarrow !) \\
\frac{\Delta \rightarrow B}{! A, \Delta \rightarrow B}(\text { weak }) & \frac{! A, ! A, \Delta \rightarrow B}{! A, \Delta \rightarrow B}(\text { contr }) \\
\frac{\Delta_{1}, B, ! A, \Delta_{2} \rightarrow C}{\Delta_{1}, ! A, B, \Delta_{2} \rightarrow C}\left(\text { perm }_{1}\right) & \frac{\Delta_{1}, ! B, A, \Delta_{2} \rightarrow C}{\Delta_{1}, A, ! B, \Delta_{2} \rightarrow C}\left(\text { perm }_{2}\right)
\end{array}
$$

The following theorem is proved in [9] and [10] and summarized in [11]. A weaker result that EL* with the product and two divisions is undecidable follows from [12, 13].

Theorem 1. The derivability problem for $\mathbf{E L}^{*}$ is undecidable.

\subsection{Lambek's Restriction}

The original Lambek calculus L [1] differs from the presented above in one detail: in $\mathbf{L}$, sequents with empty antecedents are not permitted. This restriction applies not only to the final sequent, but to all ones in the derivation. Thus, for example, the sequent $(q \backslash q) \backslash p \rightarrow p$ is derivable in $\mathbf{L}^{*}$, but not in $\mathbf{L}$, though its antecedent is not empty (but the $\mathbf{L}^{*}$-derivation involves the sequent $\rightarrow q \backslash q$ with an empty antecedent). Further we shall use the term Lambek's restriction for this special constraint. Actually, Lambek's restriction in $\mathbf{L}^{*}$ could potentially be violated only by application of the $(\rightarrow \backslash)$ and $(\rightarrow /)$ rules, therefore $\mathbf{L}$ can be obtained from $\mathbf{L}^{*}$ by adding the constraint " $\Pi$ is non-empty" to these two rules. 
At first glance, Lambek's restriction looks strange and formal, but it is highly motivated by linguistic applications.

Example 1. [4, 2.5] In syntactic formalisms based on the Lambek calculus, Lambek types denote syntactic categories. Let $n$ stand for "noun phrase," then $n / n$ is going to be a "noun modifier" (it can be combined with a noun phrase on the right producing a new, more complex noun phrase: $\mathbf{L} \vdash n / n, n \rightarrow n)$, i.e. an adjective. Adverbs, as adjective modifiers, receive the type $(n / n) /(n / n)$. Now one can derive the sequent $(n / n) /(n / n), n / n, n \rightarrow n$ and therefore establish that, say, "very interesting book" is a valid noun phrase (belongs to syntactic category $n$ ). However, in $\mathbf{L}^{*}$ one can also derive $(n / n) /(n / n), n \rightarrow n$, where the antecedent describes syntactic constructions like "very book," that in fact are not correct noun phrases.

This example shows that, for linguistic purposes, $\mathbf{L}$ is more appropriate than $\mathbf{L}^{*}$.

Suprisingly, however, it is not so straightforward to add the exponential to $\mathbf{L}$ or to impose Lambek's restriction on EL*. In Sections 2-7 we discuss several ways how to do this, define a number of the corresponding calculi, prove their properties, and discuss some issues connected with these calculi.

In Section 9 we state and prove undecidability results for calculi defined earlier; in Section 8 we prepare the techniques then used in Section 9. Finally, Section 10 contains general discussion of the results and possible directions of future work.

\subsection{Is it Possible to Maintain Three Properties Together: Lambek's Restric-} tion, Cut Elimination, and Substitution?

No. We show (Theorems 3 and 4) that for any system equipped a reasonable ! the following holds: if the system enjoys cut elimination and substitution in full extent, then this system necessarily violates Lambek's restriction. (More precisely, adding one formula starting with ! to the antecedent allows $\mathbf{L}^{*}$ derivations inside such a system.)

Nevertheless, any two of these three properties are realisable in the calculi defined below, namely:

- $\mathbf{E L}^{-}$(Section 3) has Lambek's restriction and cut elimination, but substitution only for formulae without !;

- $\mathbf{E L}^{\mathrm{mk}}$ (Section 6) has Lambek's restriction and substitution in the full form, but the cut rule is admissible only for formulae without !; 
- finally, EL* enjoys both substitution and cut, but without Lambek's restriction.

\section{Imposing Lambek's Restriction on EL*: the 1st Approach, EL ${ }^{\text {wk }}$}

The first, naïve way of imposing Lambek's restriction on EL* is to restrict only rules $(\rightarrow \backslash)$ and $(\rightarrow /)$ in the same way as it is done in $\mathbf{L}$. Notice that all other rules, including rules for the exponential, preserve the non-emptiness of the antecedent. Denote the calculus by $\mathbf{E L}^{\mathrm{wk}}$.

However, such a restriction does not change things significantly, since the following lemma provides the non-emptiness of the antecedent for free:

Lemma 2.1. Let $p$ be a variable not occurring in a sequent $\Gamma \rightarrow A$. Then

$$
\mathbf{E L}^{*} \vdash \Gamma \rightarrow A \Longleftrightarrow \mathbf{E L}^{\mathrm{wk}} \vdash ! p, \Gamma \rightarrow A .
$$

This lemma shows that $\mathbf{E L} \mathbf{L}^{*}$-derivations can be enabled in $\mathbf{E} \mathbf{L}^{\mathrm{wk}}$ by an easy technical trick. Therefore, Theorem 1 implies immediately that $\mathbf{E L}^{\text {wk }}$ is undecidable.

Lemma 2.2. $\mathbf{E L}^{\mathrm{wk}} \vdash ! B, \Gamma \rightarrow A \Longleftrightarrow \mathbf{E L}^{*} \vdash ! B, \Gamma \rightarrow A$.

These two lemmas are proved by induction on the derivations (recall that (cut) is not included in the calculi).

Thus, Lambek's restriction in $\mathbf{E L}^{\mathrm{wk}}$ vanishes as soon as the antecedent contains a formula with ! as the main connective. And, unfortunately, this acts non-locally: once! $A$ appears somewhere in the antecedent, one can freely derive unwanted things like "very book" (see Example 1 above).

\section{Imposing Lambek's Restriction on EL*: the 2nd Approach, $\mathrm{EL}^{-}$}

To overcome the ability of $! B$ to mimic the empty antecedent, we impose more radical restrictions by constructing the following calculus $\mathbf{E L}^{-}$.

Any formula not of the form $! B$ is called a non-bang-formula. (A nonbang-formula is allowed to have proper subformulae with !.) Now $\mathbf{E L}^{-}$is defined by the following axioms and rules:

$$
A \rightarrow A
$$




$$
\begin{gathered}
\frac{A, \Pi \rightarrow B}{\Pi \rightarrow A \backslash B}(\rightarrow \backslash) \text {, where } \Pi \text { contains a non-bang-formula } \\
\frac{\Pi, A \rightarrow B}{\Pi \rightarrow B / A}(\rightarrow /) \text {, where } \Pi \text { contains a non-bang-formula } \\
\frac{\Pi \rightarrow A \quad \Delta_{1}, B, \Delta_{2} \rightarrow C}{\Delta_{1}, \Pi, A \backslash B, \Delta_{2} \rightarrow C}(\backslash \rightarrow) \quad \frac{\Pi \rightarrow A \quad \Delta_{1}, B, \Delta_{2} \rightarrow C}{\Delta_{1}, B / A, \Pi, \Delta_{2} \rightarrow C}(/ \rightarrow) \\
\frac{\Delta_{1}, A, \Delta_{2} \rightarrow B}{\Delta_{1}, ! A, \Delta_{2} \rightarrow B}(! \rightarrow), \text { where } \Delta_{1}, \Delta_{2} \text { contains a non-bang-formula } \\
\frac{\Delta \rightarrow B}{! A, \Delta \rightarrow B}(\text { weak }) \quad \frac{! A, ! A, \Delta \rightarrow B}{! A, \Delta \rightarrow B}(\text { contr }) \\
\frac{\Delta_{1}, B, ! A, \Delta_{2} \rightarrow C}{\Delta_{1}, ! A, B, \Delta_{2} \rightarrow C}\left(\text { perm }{ }_{1}\right) \quad \frac{\Delta_{1}, ! B, A, \Delta_{2} \rightarrow C}{\Delta_{1}, A, ! B, \Delta_{2} \rightarrow C}\left(\text { perm }{ }_{2}\right)
\end{gathered}
$$

Note that in the $\left(\rightarrow\right.$ !) rule of $\mathbf{E L}^{*}$ all the formulae in the antecedent are of the form !B. Therefore there is no $(\rightarrow !)$ rule in $\mathbf{E L}^{-}$. Also note that the cut rule is not officially included in $\mathbf{E L}^{-}$; in the next section we prove that it is admissible.

Lemma 3.1. If $\Pi \rightarrow A$ is derivable in $\mathbf{E L}^{-}$and $\Pi$ is of the form $! \Gamma$, then $A$ is of the form !B, such that $! B$ appears in $! \Gamma$.

Proof. A sequent with no non-bang-formula in the antecedent can be obtained only by applying (weak), (perm), (contr) (in any combination) to an axiom of the form $! B \rightarrow ! B$. All other rules either introduce non-bangformulae to the left, or explicitly require their existence.

Now Lambek's restriction in $\mathbf{E L}^{-}$is stated in the following way: in a non-trivial derivable sequent $\Pi \rightarrow A$ the antecedent $\Pi$ should contain at least one non-bang-formula. 


\section{The Cut Rule in $\mathrm{EL}^{-}$}

Lemma 4.1. If $\Pi \rightarrow A$ is derivable in $\mathbf{E L}^{-}$and $A$ is a non-bang-formula, then $\Pi$ necessarily contains a non-bang-formula.

Proof. Immediately from Lemma 3.1.

Theorem 2. The cut rule

$$
\frac{\Pi \rightarrow A \quad \Delta_{1}, A, \Delta_{2} \rightarrow C}{\Delta_{1}, \Pi, \Delta_{2} \rightarrow C}(\mathrm{cut})
$$

is admissible in $\mathbf{E L}^{-}$.

ProOf. We proceed by double induction. We consider a number of cases, and in each of them the cut either disappears, or is replaced by cuts with simpler cut formulae $(A)$, or is replaced by a cut for which the depth of at least one derivation tree of a premise $\left(\Pi \rightarrow A\right.$ or $\left.\Delta_{1}, B, \Delta_{2} \rightarrow C\right)$ is less than for the original cut, and the other premise derivation and the cut formula remain the same. Thus by double induction (on the outer level-on the complexity of $A$, on the inner level - on the sum of premise derivation tree depths) we get rid of the cut.

Case 1: One of the premises of the cut rule is the axiom (a sequent of the form $A \rightarrow A$ ). Then the cut disappears, since its other premise concides with the goal sequent.

Case 2: $A$ is not the formula that is introduced by the lowermost rule in the derivation of one of the premises of the cut. (The term "formula introduced by a rule" here means the following: rules $(/ \rightarrow),(\backslash \rightarrow),(\rightarrow /),(\rightarrow \backslash)$, $(! \rightarrow)$, and $(\rightarrow !)$ introduce the formula that includes the new connective; (weak) and (contr) introduce $! A$ involved in these rules; $\left(\right.$ perm $\left._{1}\right)$ and $\left(\right.$ perm $\left._{2}\right)$ do not introduce anything.)

In this case (cut) can be interchanged with that lowermost rule. Many subcases arise here, depending on the particular form of the rule interchanged with (cut), but they are all handled similarly. Below we show only the most interesting situations, when we interchange $(! \rightarrow)$ or $(\rightarrow /)((\rightarrow \backslash)$ is symmetric) with (cut). In these transformations Lambek's restriction imposed on these rules could potentially get violated after the exchange with (cut) (we show that this does not happen). 
Transformation 1.

$$
\begin{aligned}
& \frac{\frac{\Pi^{\prime}, D, \Pi^{\prime \prime} \rightarrow A}{\Pi^{\prime}, ! D, \Pi^{\prime \prime} \rightarrow A}(! \rightarrow) \quad \Delta_{1}, A, \Delta_{2} \rightarrow C}{\Delta_{1}, \Pi^{\prime}, ! D, \Pi^{\prime \prime}, \Delta_{2} \rightarrow C}(\mathrm{cut}) \\
& 3 \\
& \frac{\Pi^{\prime}, D, \Pi^{\prime \prime} \rightarrow A \quad \Delta_{1}, A, \Delta_{2} \rightarrow C}{\frac{\Delta_{1}, \Pi^{\prime}, D, \Pi^{\prime \prime}, \Delta_{2} \rightarrow C}{\Delta_{1}, \Pi^{\prime}, ! D, \Pi^{\prime \prime}, \Delta_{2} \rightarrow C}(! \rightarrow)}(\text { cut })
\end{aligned}
$$

Transformation 2.

$$
\begin{aligned}
& \frac{\Pi \rightarrow A \frac{\Delta_{1}, A, \Delta_{2}^{\prime}, D, \Delta_{2}^{\prime \prime} \rightarrow C}{\Delta_{1}, A, \Delta_{2}^{\prime}, ! D, \Delta_{2}^{\prime \prime} \rightarrow C}}{\Delta_{1}, \Pi, \Delta_{2}^{\prime}, ! D, \Delta_{2}^{\prime \prime} \rightarrow C}\left(\begin{array}{l}
\text { cut }) \\
\text { ( })
\end{array}\right. \\
& 3 \\
& \frac{\Pi \rightarrow A \quad \Delta_{1}, A, \Delta_{2}^{\prime}, D, \Delta_{2}^{\prime \prime} \rightarrow C}{\frac{\Delta_{1}, \Pi, \Delta_{2}^{\prime}, D, \Delta_{2}^{\prime \prime} \rightarrow C}{\Delta_{1}, \Pi, \Delta_{2}^{\prime}, ! D, \Delta_{2}^{\prime \prime} \rightarrow C}(! \rightarrow)}(\text { cut })
\end{aligned}
$$

Transformation 3.

$$
\begin{gathered}
\frac{\Delta_{1}, A, \Delta_{2}, C_{1} \rightarrow C_{2}}{\Delta_{1}, A, \Delta_{2} \rightarrow C_{2} / C_{1}}(\rightarrow /) \\
\text { (cut) } \\
\Delta_{1}, \Pi, \Delta_{2} \rightarrow C_{2} / C_{1} \\
\frac{\Pi \rightarrow A \quad \Delta_{1}, A, \Delta_{2}, C_{1} \rightarrow C_{2}}{\Delta_{1}, \Pi, \Delta_{2}, C_{1} \rightarrow C_{2}}(\text { cut }) \\
\frac{\Delta_{1}, \Pi, \Delta_{2} \rightarrow C_{2} / C_{1}}{\Delta_{1}} \text { ) }
\end{gathered}
$$

In Transformation 1, the existence of a non-bang-formula in $\Pi^{\prime}$ or $\Pi^{\prime \prime}$ implies its existence in the larger context $\Delta_{1}, \Pi^{\prime}, \Pi^{\prime \prime}, \Delta_{2}$, thus application of $(! \rightarrow)$ is legal. For Transformations 2 and 3, if the non-bang-formula guaranteed by Lambek's restriction (which was indeed valid before the transformation) is $A$ itself, then a non-bang-formula also appears in $\Pi$ by Lemma 4.1 . 
Otherwise we can take the same non-bang-formula as before the transformation.

Case 3: $A$ is introduced by the lowermost rules both into $\Pi \rightarrow A$ and into $\Delta_{1}, A, \Delta_{2} \rightarrow C$. Note that in $\mathbf{E L}^{-}$there is no rule that introduces a formula of the form ! $E$ to the succedent. Therefore $A$ is either of the form $E / F$, or $F \backslash E$. We consider only the former, the latter is handled symmetrically. The derivation is transformed in the following way:

Transformation 4.

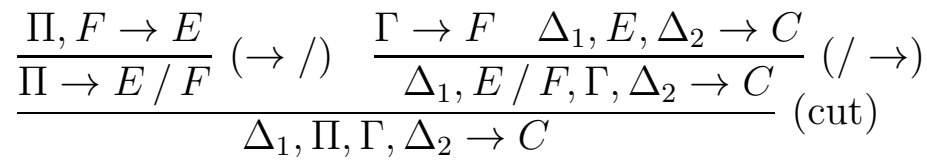

$$
\begin{aligned}
& \xi \\
& \frac{\Gamma \rightarrow F \quad \Pi, F \rightarrow E}{\frac{\Pi, \Gamma \rightarrow E}{(\text { cut }) \quad \Delta_{1}, E, \Delta_{2} \rightarrow C}} \text { (cut) }
\end{aligned}
$$

The new cut formulae, $E$ and $F$, are simpler than the original one, $E / F$.

\section{Substitution Issues}

$\mathbf{E L}^{-}$inherits a bang-free substitution lemma: derivability of a sequent is preserved if we replace all occurrences of a variable $q$ with a formula $Q$ without!.

In the general case, however, $\mathbf{E L}^{-}$does not respect type substitution. For instance, $p, !(p \backslash q) \rightarrow q$ is derivable in $\mathbf{E L}^{-}$, but $! r, !(! r \backslash q) \rightarrow q$ is not. Unfortunately, this is not just a problem with this particular system, but a general issue: as we show below, in the presence of the exponential modality any system enjoying admissibility of (cut) and general substitution lemma necessarily violates Lambek's restriction.

Let $\mathbf{E L}^{\dagger}$ be an arbitrary calculus, in the same language as $\mathbf{E L}^{*}$, satisfying the properties below. (Note that these properties do not define the calculus in a unique way - we rather talk about a family of possible 'good' extensions of the Lambek calculus.)

1. Extension. If a sequent is derivable in $\mathbf{L}$, then it is derivable in $\mathbf{E L}^{\dagger}$. 
2. Cut. The cut rule of the form

$$
\frac{\Pi \rightarrow A \quad \Delta_{1}, A, \Delta_{2} \rightarrow C}{\Delta_{1}, \Pi, \Delta_{2} \rightarrow C}(\text { cut })
$$

is admissible in $\mathbf{E L}^{\dagger}$.

3. Substitution. The following rule is admissible in $\mathbf{E} \mathbf{L}^{\dagger}$ :

$$
\frac{\Pi \rightarrow A}{\Pi[q:=Q] \rightarrow A[q:=Q]} \text { (subst) }
$$

Here $q$ is a variable, $Q$ is a formula (possibly with !), and $[q:=Q]$ denotes substitution of $Q$ for $q$.

4. Monotonicity. The following rules are admissible in $\mathbf{E L}^{\dagger}$ :

$$
\begin{aligned}
& \frac{A_{1} \rightarrow A_{2} \quad B_{1} \rightarrow B_{2}}{B_{1} / A_{2} \rightarrow B_{2} / A_{1}}\left(\mathrm{mon}_{/}\right) \\
& \frac{A_{1} \rightarrow A_{2} \quad B_{1} \rightarrow B_{2}}{A_{2} \backslash B_{1} \rightarrow A_{1} \backslash B_{2}}\left(\text { mon }_{\backslash}\right)
\end{aligned}
$$

5. Weakening, contraction, and permutation. The following rules are admissible in $\mathbf{E L}^{\dagger}$ :

$$
\begin{array}{cc}
\frac{\Delta \rightarrow B}{! A, \Delta \rightarrow B} \text { (weak) } & \frac{! A, ! A, \Delta \rightarrow B}{! A, \Delta \rightarrow B} \text { (contr) } \\
\frac{\Delta_{1}, B, ! A, \Delta_{2} \rightarrow C}{\Delta_{1}, ! A, B, \Delta_{2} \rightarrow C}\left(\text { perm }_{1}\right) & \frac{\Delta_{1}, ! B, A, \Delta_{2} \rightarrow C}{\Delta_{1}, A, ! B, \Delta_{2} \rightarrow C}\left(\text { perm }_{2}\right)
\end{array}
$$

6. The rules

$$
\frac{\Pi \rightarrow A \quad \Delta_{1}, B, \Delta_{2} \rightarrow C}{\Delta_{1}, B / A, \Pi, \Delta_{2} \rightarrow C}(/ \rightarrow) \quad \text { and } \quad \frac{\Pi \rightarrow A \quad \Delta_{1}, B, \Delta_{2} \rightarrow C}{\Delta_{1}, \Pi, A \backslash B, \Delta_{2} \rightarrow C}(\backslash \rightarrow)
$$

are admissible in $\mathbf{E L}^{\dagger}$ without restrictions.

7. If $\Pi$ contains a formula without occurrences of ! (and therefore is nonempty) and $B$ does not contain occurrences of !, then the rules

$$
\frac{\Pi, A \rightarrow B}{\Pi \rightarrow B / A}(\rightarrow /) \quad \text { and } \quad \frac{A, \Pi \rightarrow B}{\Pi \rightarrow A \backslash B}(\rightarrow \backslash)
$$

are admissible in $\mathbf{E L}^{\dagger}$. 
Note that (cut), (subst), and (mon) are admissible in $\mathbf{L}$, therefore we want them to keep valid in the extension. Weakening, contraction, and permutation are basic rules for the exponential. Finally, the last two properties ensure that the version of Lambek's restriction used in $\mathbf{E L}^{\dagger}$ does not forbid Lambek derivations in the presence of the exponential modality.

Also note that by substitution we get the axiom $A \rightarrow A$ for arbitrary $A$, possibly with occurrences of !.

Unfortunately, any calculus $\mathbf{E L}^{\dagger}$ with these 7 properties necessarily violates Lambek's restriction:

Lemma 5.1. If $\mathbf{E L}^{\dagger}$ satisfies properties 1-7, $A$ and $B$ do not contain !, and $\mathbf{E L}^{\dagger} \vdash ! q, A \rightarrow B$, then $\mathbf{E L}^{\dagger} \vdash ! q \rightarrow A \backslash B$ and $\mathbf{E L}^{\dagger} \vdash ! q \rightarrow B / A$.

ProOF.

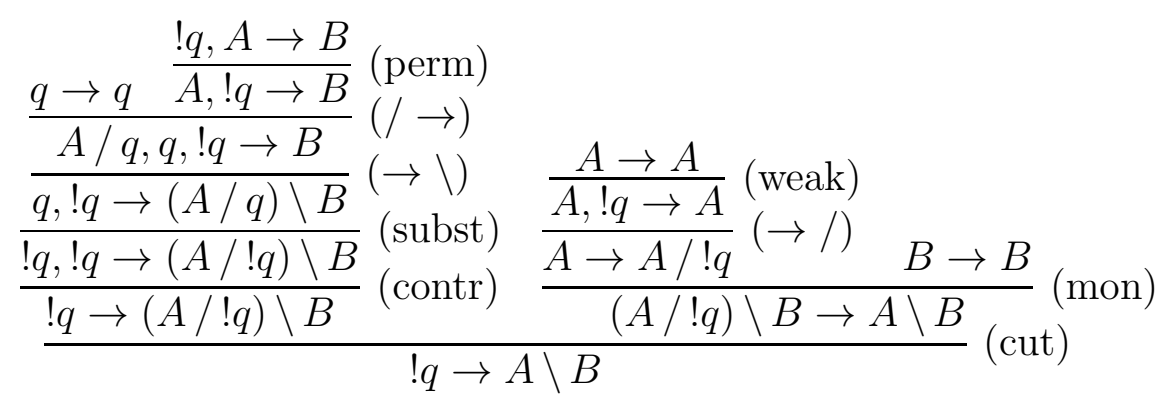

The / case is symmetric.

Theorem 3. If $\Pi \rightarrow B$ is derivable in $\mathbf{L}^{*}$, and $\mathbf{E L}^{\dagger}$ satisfies properties 1- 7 , then $! q, \Pi \rightarrow B$ is derivable in $\mathbf{E L}^{\dagger}$.

Proof. Induction on derivation length. For the axiom case we use the weakening rule to add ! $q$. Applications of Lambek rules are translated straightforwardly; the only non-trivial case is $(\rightarrow /)$ and $(\rightarrow \backslash)$ with an empty $\Pi$, where we use Lemma 5.1.

One could think that this effect is due to the weakening rule (this rule allows forcing the antecedent to be non-empty). However, in the fragment with only one variable a result like Theorem 3 can be achieved without weakening. Note that, in the view of [14], [15], [16, Chapter 3], [17], and [18], the onevariable fragment of the Lambek calculus is as powerful as the full calculus with a countable set of variables. 
Theorem 4. Let $\mathbf{E L}^{\ddagger}$ satisfy properties 1-4, 6, and \%. Let also contraction and permutation (but not weakening) rules be admissible in $\mathbf{E L}^{\ddagger}$, and, in addition, let $\mathbf{E L}^{\ddagger}$ include the $(! \rightarrow)$ rule of the form

$$
\frac{\Delta_{1}, A, \Delta_{2} \rightarrow C}{\Delta_{1}, ! A, \Delta_{2} \rightarrow C}(! \rightarrow)
$$

if $\Delta_{1}$ or $\Delta_{2}$ contains a formula without occurrences of ! (this is the strongest version of Lambek's restriction that could be imposed on this rule). In this case, if $\Pi$ and $B$ contain only one variable $p$ and do not contain occurrences of $!$, and $\Pi \rightarrow B$ is derivable in $\mathbf{L}^{*}$, then $\Pi, !(p \backslash p) \rightarrow B$ is derivable in $\mathbf{E L}^{\ddagger}$.

Proof. First we state an easy technical lemma:

Lemma 5.2. If $A$ contains only one variable $p$ and no occurrences of !, then $\mathbf{E L}^{\ddagger} \vdash A, !(p \backslash p) \rightarrow A$.

Proof. Induction on the complexity of $A$. If $A=p$, then $p, !(p \backslash p) \rightarrow p$ is derived as follows:

$$
\begin{gathered}
\frac{p \rightarrow p \quad p \rightarrow p}{p, p \backslash p \rightarrow p} \\
\frac{p, !(p \backslash p) \rightarrow p}{(}(! \rightarrow)
\end{gathered}
$$

For $A=A_{2} / A_{1}$ and $A=A_{1} \backslash A_{2}$ we use the following derivations:

$$
\begin{array}{ll}
\frac{A_{1} \rightarrow A_{1} \quad A_{2}, !(p \backslash p) \rightarrow A_{2}}{A_{2} / A_{1}, A_{1}, !(p \backslash p) \rightarrow A_{2}} & (/ \rightarrow) \\
\frac{A_{2} / A_{1}, !(p \backslash p), A_{1} \rightarrow A_{2}}{A_{2} / A_{1}, !(p \backslash p) \rightarrow A_{2} / A_{1}} & (\rightarrow /)
\end{array} \quad \frac{A_{1} \rightarrow A_{1} \quad A_{2}, !(p \backslash p) \rightarrow A_{2}}{\frac{A_{1}, A_{1} \backslash A_{2}, !(p \backslash p) \rightarrow A_{2}}{A_{1} \backslash A_{2}, !(p \backslash p) \rightarrow A_{1} \backslash A_{2}}(\rightarrow \backslash)}(\rightarrow)
$$

$A_{1} \rightarrow A_{1}$ is an axiom of $\mathbf{L}$ (and, by property 1 , is derivable in $\mathbf{E L}^{\ddagger}$ ). $A_{2}, !(p \backslash p) \rightarrow A_{2}$ is derivable by induction hypothesis.

Then we proceed by induction on derivation. The axiom $A \rightarrow A$ becomes a derivable sequent $A, !(p \backslash p) \rightarrow A$ (Lemma 5.2). Now the only non-trivial case is to simulate $(\rightarrow /)$ and $(\rightarrow \backslash)$ with an empty $\Pi$ :

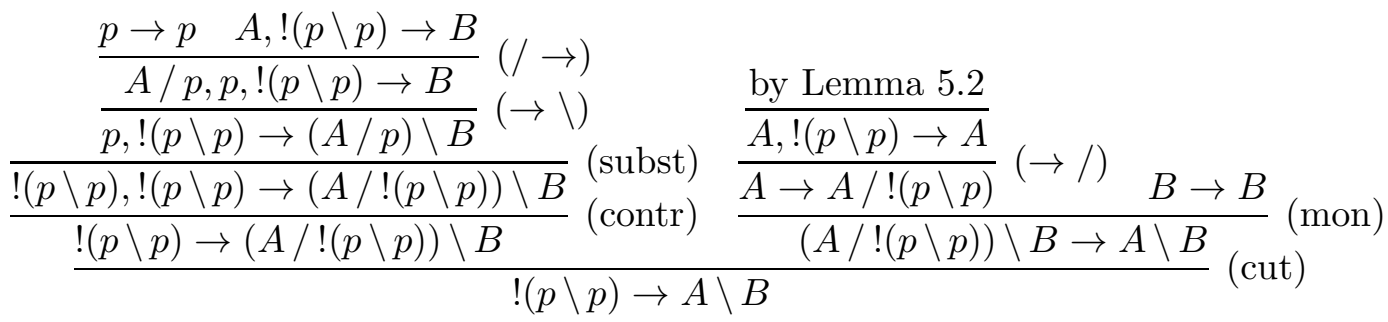


The $(\rightarrow /)$ case is handled symmetrically.

Theorems 3 and 4 show that there is no way to add the exponential modality to the Lambek calculus preserving Lambek's restriction, admissibility of (cut), and the substitution property at the same time.

In the next section we describe another yet extension of $\mathbf{L}$ with the exponential modality. This extension features a version Lambek's restriction, admits substitution of formulae with !, but, on the other hand, only a limited version of the cut rule.

\section{The 3rd Approach: EL ${ }^{\mathrm{mk}}$}

In order to restore type substitution as much as possible we consider the third approach to imposing Lambek's restriction on EL*. The trade-off here is that the cut rule is going to be admissible only in a limited form.

We present such a system in the form of marked sequent calculus. A marked sequent is an expression of the form $\Pi \rightarrow A$, where $A$ is a type and $\Pi$ is a sequence of pairs of the form $\langle B, m\rangle$, written as $B_{(m)}$, where $B$ is a type and $m \in\{0,1\}$ is the marking bit. A pair $B_{(0)}$ is called an unmarked type, and $B_{(1)}$ is called a marked type. The marking bits are utilized inside the derivation, and in the end they are forgotten, yielding a sequent in the original sense. If $\Gamma=B_{1\left(m_{1}\right)}, \ldots, B_{k\left(m_{k}\right)}$, then by ! $\Gamma$ we denote the sequence $\left(! B_{1}\right)_{\left(m_{1}\right)}, \ldots,\left(! B_{k}\right)_{\left(m_{k}\right)}$.

Lambek's restriction is now formulated as follows: every sequent should contain an unmarked type in the antecedent.

The calculus $\mathbf{E L}^{\mathrm{mk}}$ is defined in the following way:

$$
\begin{gathered}
p_{(0)} \rightarrow p \\
\frac{\Pi, A_{(m)} \rightarrow B}{\Pi \rightarrow B / A}(\rightarrow /) \text {, where } \Pi \text { contains an unmarked type } \\
\frac{A_{(m)}, \Pi \rightarrow B}{\Pi \rightarrow A \backslash B}(\rightarrow \backslash), \text { where } \Pi \text { contains an unmarked type } \\
\frac{\Pi \rightarrow A \Delta_{1}, B_{(m)}, \Delta_{2} \rightarrow C}{\Delta_{1},(B / A)_{(m)}, \Pi, \Delta_{2} \rightarrow C}(/ \rightarrow) \quad \frac{\Pi \rightarrow A \quad \Delta_{1}, B_{(m)}, \Delta_{2} \rightarrow C}{\Delta_{1}, \Pi,(A \backslash B)_{(m)}, \Delta_{2} \rightarrow C}(\backslash \rightarrow)
\end{gathered}
$$




$$
\begin{gathered}
\frac{\Delta_{1}, A_{(m)}, \Delta_{2} \rightarrow B}{\Delta_{1},(! A)_{(1)}, \Delta_{2} \rightarrow B}(! \rightarrow), \text { where } \Delta_{1}, \Delta_{2} \text { contains an unmarked type } \\
\frac{! \Gamma, \Delta \rightarrow A}{! \Gamma, ! \Delta \rightarrow ! A}(\rightarrow !) \quad \frac{\Delta_{1}, \Delta_{2} \rightarrow A}{\Delta_{1},(! A)_{(1)}, \Delta_{2} \rightarrow A} \text { (weak) } \\
\frac{(! A)_{\left(m_{1}\right)},(! A)_{\left(m_{2}\right)}, \Delta \rightarrow B}{(! A)_{\left(\min \left\{m_{1}, m_{2}\right\}\right)}, \Delta \rightarrow B}(\text { contr }) \\
\frac{\Delta_{1}, B_{\left(m_{2}\right)},(! A)_{\left(m_{1}\right)}, \Delta_{2} \rightarrow C}{\Delta_{1},(! A)_{\left(m_{1}\right)}, B_{\left(m_{2}\right)}, \Delta_{2} \rightarrow C}\left(\operatorname{perm}_{1}\right) \quad \frac{\Delta_{1},(! B)_{\left(m_{2}\right)}, A_{\left(m_{1}\right)}, \Delta_{2} \rightarrow C}{\Delta_{1}, A_{\left(m_{1}\right)},(! B)_{\left(m_{2}\right)}, \Delta_{2} \rightarrow C}\left(\text { perm }_{2}\right)
\end{gathered}
$$

Recall that all proofs are cut-free. Also note that in EL we use a stronger form of the $(\rightarrow !)$ rule. In $\mathbf{E L}^{*}$ this new rule could be simulated by applying the $(! \rightarrow)$ rule for all formulae in $\Delta$ and then using the original $(\rightarrow !)$ rule, but here the $(! \rightarrow)$ rule will fail to satisfy the restriction.

The substitution property is now formulated as follows:

Theorem 5. Let $A[q:=Q]$ (resp., $\Pi[q:=Q]$ ) be the result of substituting $Q$ for $q$ in type $A$ (resp., marked sequence $\Pi$ ). Then $\mathbf{E L}^{\mathrm{mk}} \vdash \Pi \rightarrow A$ implies $\mathbf{E L}^{\mathrm{mk}} \vdash \Pi[q:=Q] \rightarrow A[q:=Q]$.

Proof. By structural induction on $Q$ we prove that $\mathbf{E L}^{\mathrm{mk}} \vdash Q_{(0)} \rightarrow Q$ for every type $Q$. Then we just replace $q$ with $Q$ everywhere in the proof.

The cut rule in $\mathbf{E L}^{\mathrm{mk}}$ is generally not admissible: the sequents $(! q)_{(0)} \rightarrow$ $(p / ! q) \backslash p$ and $((p / ! q) \backslash p)_{(0)} \rightarrow p \backslash p$ are derivable in $\mathbf{E L}^{\mathrm{mk}}$, but $(! q)_{(0)} \rightarrow$ $p \backslash p$ is not. This counterexample is actually taken from the proof of Theorem 3 ,

The cut rule is admissible only in the following limited version:

Theorem 6. If $\mathbf{E L}^{\mathrm{mk}} \vdash \Pi \rightarrow A$, $\mathbf{E L}^{\mathrm{mk}} \vdash \Delta_{1}, A_{(0)}, \Delta_{2} \rightarrow C$, and $A$ does not contain!, then $\mathbf{E L}^{\mathrm{mk}} \vdash \Delta_{1}, \Pi, \Delta_{2} \rightarrow C$. 
This theorem is proved using the standard argument, just as for $\mathbf{L}$.

Compare $\mathbf{E L}^{\mathrm{mk}}$ with $\mathbf{E L}^{-}$. These two systems are not connected with any strong form of conservativity or equivalence: on one hand, the sequent $! r, r \backslash ! p, !(p \backslash q) \rightarrow q$ is derivable in $\mathbf{E L}-$, but not in $\mathbf{E} \mathbf{L}^{\mathrm{mk}}$; on the other hand, for $! p, !(! p \backslash q) \rightarrow q$ the situation is opposite. Fortunately, the following holds:

Lemma 6.1. If $\Gamma$, $\Pi$, and $A$ do not contain!, then

$$
\mathbf{E L}^{\mathrm{mk}} \vdash ! \Gamma, \Pi \rightarrow A \Longleftrightarrow \mathbf{E L}^{-} \vdash ! \Gamma, \Pi \rightarrow A .
$$

Proof. Since for a sequent of the form $! \Gamma, \Pi \rightarrow A$ the rule $(\rightarrow !)$ can never appear in the proof, marked types in the antecedent are exactly the types starting with !, and the two versions of Lambek's restriction coincide.

\section{Conservativity over L}

The three calculi defined above are conservative over $\mathbf{L}$ :

Proposition 7. If $\Pi$ and $A$ do not contain!, then

$\mathbf{L} \vdash \Pi \rightarrow A \Longleftrightarrow \mathbf{E L}^{\mathrm{wk}} \vdash \Pi \rightarrow A \Longleftrightarrow \mathbf{E L}^{-} \vdash \Pi \rightarrow A \Longleftrightarrow \mathbf{E L}^{\mathrm{mk}} \vdash \Pi \rightarrow A$

(for $\mathbf{E} \mathbf{L}^{\mathrm{mk}}$, all types in $\Pi$ get the 0 marking bit).

Note that $\Pi$ is necessarily non-empty.

Therefore, we guarantee that in all approaches the innovation affects only the new exponential connective, and keeps the original Lambek system intact. For $\mathbf{E L}^{-}$and $\mathbf{E L}{ }^{\mathrm{mk}}$ adding fresh exponentials to the antecedent also does not affect Lambek's restriction:

Proposition 8. If $\Pi$ and $A$ do not contain!, and $p$ is a variable not occurring in $\Pi$ and $A$, then

$$
\mathbf{E L}^{-} \vdash ! p, \Pi \rightarrow A \Longleftrightarrow \mathbf{E L}^{\mathrm{mk}} \vdash ! p, \Pi \rightarrow A \Longleftrightarrow \mathbf{L} \vdash \Pi \rightarrow A
$$

(for the $\mathbf{E L}^{\mathrm{mk}}$ case, !p gets marking bit 1 and types from $\Pi$ get 0 ).

For $\mathbf{E L}^{\mathrm{wk}}$, due to Lemma 2.1, the situation is different: if $\Pi$ and $A$ do not contain !, and $p$ is a fresh variable, then

$$
\mathbf{E L}^{\mathrm{wk}} \vdash ! p, \Pi \rightarrow A \Longleftrightarrow \mathbf{E L}^{*} \vdash \Pi \rightarrow A \Longleftrightarrow \mathbf{L}^{*} \vdash \Pi \rightarrow A .
$$

Recall that, for example, $(q \backslash q) \backslash p \rightarrow p$ is derivable in $\mathbf{L}^{*}$, but not in $\mathbf{L}$. 


\section{Generative Grammars and the Lambek Calculus with Non-Logical Axioms}

In this subsection we introduce axiomatic extensions of the Lambek calculus $\mathbf{L}$, following [19]. These extensions are going to be useful for proving undecidability results à la Theorem 1 .

Let $\mathcal{A}$ be a set of sequents. Then by $\mathbf{L}+\mathcal{A}$ we denote $\mathbf{L}$ augmented with sequents from $\mathcal{A}$ as new axioms and also the cut rule (which is no longer eliminable). Elements of $\mathcal{A}$ are called non-logical axioms.

Further we consider non-logical axioms of a special form: either $p, q \rightarrow r$, or $p / q \rightarrow r$, where $p, q, r$ are variables. Buszkowski calls them special nonlogical axioms. In this case, $\mathbf{L}+\mathcal{A}$ can be formulated in a cut-free way [19]: instead of non-logical axioms of the form $p, q \rightarrow r$ or $p / q \rightarrow r$ we use rules

$$
\frac{\Pi_{1} \rightarrow p \quad \Pi_{2} \rightarrow q}{\Pi_{1}, \Pi_{2} \rightarrow r}\left(\operatorname{red}_{1}\right) \quad \text { and } \quad \frac{\Pi, q \rightarrow p}{\Pi \rightarrow r}\left(\operatorname{red}_{2}\right) \text {, where } \Pi \neq \Lambda
$$

respectively. This calculus admits the cut rule [19]. Further we'll mean it when talking about $\mathbf{L}+\mathcal{A}$. We'll use the term Buszkowski's rules for $\left(\operatorname{red}_{i}\right)$.

Now we define two notions of formal grammar. The first one is the widely known formalism of generative grammars introduced by Chomsky. If $\Sigma$ is an alphabet (i.e. a finite non-empty set), then by $\Sigma^{*}$ we denote the set of all words over $\Sigma$ (including the empty word). A generative grammar is a quadruple $G=\langle N, \Sigma, s, P\rangle$, where $N$ and $\Sigma$ are two disjoint alphabets, $s \in N$, and $P$ is a set or rules. Here we consider only rules of two forms: $x \rightarrow y_{1} y_{2}$ or $x_{1} x_{2} \rightarrow y$, where $x, y, x_{i}, y_{i} \in N \cup \Sigma$. If $v=u_{1} \alpha u_{2}, w=u_{1} \beta u_{2}$, and $(\alpha \rightarrow \beta) \in P$, then this rule can be applied to $v$ yielding $w: v \Rightarrow w$. By $\Rightarrow^{*}$ we denote the reflexive and transitive closure of $\Rightarrow$. Finally, the language generated by $G$ is the set of all words $w \in \Sigma^{*}$, such that $s \Rightarrow^{*} w$. Note that the empty word cannot be produced by a generative grammar as defined above.

It is well known that the class of languages generated by generative grammars coincides with the class of all recursively enumerable (r. e.) languages without the empty word.

The second family of formal grammar we are going to consider is the class of Lambek categorial grammars with non-logical axioms. A Lambek grammar is a tuple $\mathcal{G}=\langle\Sigma, \mathcal{A}, H, \triangleright\rangle$, where $\Sigma$ is an alphabet, $\mathcal{A}$ is a set of non-logical axioms, $H$ is a type, and $\triangleright \subseteq \operatorname{Tp} \times \Sigma$ is a finite binary correspondence between types and letter, called type assignment. A word $w=a_{1} \ldots a_{n}$ 
belongs to the language generated by $\mathcal{G}$ iff there exist such types $A_{1}, \ldots, A_{n}$ that $A_{i} \triangleright a_{i}(i=1, \ldots, n)$ and $\mathbf{L}+\mathcal{A} \vdash A_{1}, \ldots, A_{n} \rightarrow H$.

If we use $\mathbf{L}$ / instead of $\mathbf{L}$, we get the notion of $\mathbf{L}$ /-grammar with nonlogical axioms. It's easy to see that all languages generated by Lambek grammars are r. e., therefore, they can be generated by generative grammars. Buszkowski [19] proves the converse:

Theorem 9. Every language generated by a generative grammar can be generated by an $\mathbf{L}_{/}$-grammar with special non-logical axioms.

In comparison, for $\mathcal{A}=\varnothing$ Pentus' theorem [20] states that all languages generated are context-free. Thus, even simple (special) non-logical axioms dramatically increase the power (and complexity) of Lambek grammars.

Since there exist undecidable r. e. languages, Buszkowski obtains the following [19]:

Theorem 10. There exists such $\mathcal{A}$ that the derivability problem for $\mathbf{L}_{/}+\mathcal{A}$ is undecidable.

\section{Undecidability of $\mathrm{EL}^{-}$and $\mathrm{EL}^{\mathrm{mk}}$}

Recall that $\mathbf{E L}^{-}$, defined in Section 3 , involves two division operations, and no product. The calculus $\mathbf{E L}^{-}$, is the fragment of $\mathbf{E L}{ }^{-}$, where we confine ourselves only to the right division.

Theorem 11. The derivability problem for $\mathbf{E L}^{-}$and even for $\mathbf{E L}^{-}$, is undecidable.

We take a set $\mathcal{A}$ of non-logical axioms of non-logical axioms of the forms $p, q \rightarrow r$ or $p / q \rightarrow r$ and encode them in $\mathbf{E L}^{-}$using the exponential. Let $\mathcal{G}_{\mathcal{A}}=\{(r / q) / p \mid(p, q \rightarrow r) \in \mathcal{A}\} \cup\{r /(p / q) \mid(p / q \rightarrow r) \in \mathcal{A}\}$ and let $\Gamma_{\mathcal{A}}$ be a sequence of all types from $\mathcal{G}_{\mathcal{A}}$ in any order. Then the following holds:

Lemma 9.1. $\mathbf{L} /+\mathcal{A} \vdash \Pi \rightarrow A \Longleftrightarrow \mathbf{E L}_{/}^{-} \vdash ! \Gamma_{\mathcal{A}}, \Pi \rightarrow A$.

Proof. $\Rightarrow$ Proceed by induction on the derivation of $\Pi \rightarrow A$ in $\mathbf{L}_{/}+\mathcal{A}$. If $\Pi \rightarrow A$ is an axiom of the form $A \rightarrow A$, then we get $\mathbf{E L}^{-}, \vdash ! \Gamma_{\mathcal{A}}, A \rightarrow A$ by application of the (weak) rule. 
If $A=B / C$, and $\Pi \rightarrow A$ is obtained using the $(\rightarrow /)$ rule, then $! \Gamma_{\mathcal{A}}, \Pi \rightarrow A$ is derived using the same rule:

$$
\frac{! \Gamma_{\mathcal{A}}, \Pi, C \rightarrow B}{! \Gamma_{\mathcal{A}}, \Pi \rightarrow B / C}
$$

Here $\Pi$ is not empty, and consists of non-bang-formulae, therefore the application of this rule is eligible in $\mathbf{E L}^{-} ; \mathbf{E L}^{-} / \vdash ! \Gamma_{\mathcal{A}}, \Pi, C \rightarrow B$ by induction hypothesis.

If $\Pi=\Phi_{1}, B / C, \Psi, \Phi_{2}$, and $\Pi \rightarrow A$ is obtained by $(/ \rightarrow)$ from $\Psi \rightarrow C$ and $\Phi_{1}, B, \Phi_{2} \rightarrow A$, then for $! \Gamma_{\mathcal{A}}, \Pi \rightarrow A$ we have the following derivation in $\mathbf{E L}^{-}$, where ${ }^{*}$ means several applications of the rules in any order.

$$
\begin{aligned}
& \frac{! \Gamma_{\mathcal{A}}, \Psi \rightarrow C \quad ! \Gamma_{\mathcal{A}}, \Phi_{1}, B, \Phi_{2} \rightarrow A}{! \Gamma_{\mathcal{A}}, \Phi_{1}, B / C, ! \Gamma_{\mathcal{A}}, \Psi, \Phi_{2} \rightarrow A} \\
& \frac{\frac{! \Gamma_{\mathcal{A}}, ! \Gamma_{\mathcal{A}}, \Phi_{1}, B / C, \Psi, \Phi_{2} \rightarrow A}{!}\left(\Gamma_{\mathcal{A}}, \Phi_{1}, B / C, \Psi, \Phi_{2} \rightarrow A\right.}{\left(\text { perm }_{1}\right)^{*}}\left(\text { contr }^{*} \text { perm }_{1}\right)^{*}
\end{aligned}
$$

Finally, $\Pi \rightarrow A$ can be obtained by application of Buszkowski's rules $\left(\operatorname{red}_{1}\right)$ or $\left(\operatorname{red}_{2}\right)$. In the first case, $A=r, \Pi=\Pi_{1}, \Pi_{2} ; \mathbf{L}+\mathcal{A} \vdash \Pi_{1} \rightarrow p$, and $\mathbf{L}+\mathcal{A} \vdash \Pi_{2} \rightarrow q$. Furthermore, $\mathcal{G}_{\mathcal{A}} \ni(r / q) / p$, thus we get the following derivation in $\mathbf{E L}^{-}$:

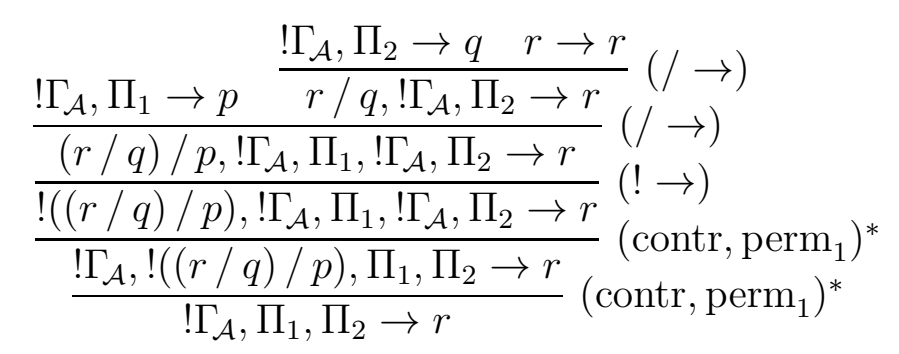

The application of $(! \rightarrow)$ here is legal, since $\Pi_{1}$ and $\Pi_{2}$ are non-empty and consist of non-bang-formulae.

In the $\left(\operatorname{red}_{2}\right)$ case, $A=r$, and we have $! \Gamma_{\mathcal{A}}, \Pi, q \rightarrow p$ in the induction hypothesis. Again, $\mathcal{G}_{\mathcal{A}} \ni r /(p / q)$, and we proceed like this:

$$
\begin{aligned}
& \frac{\frac{! \Gamma_{\mathcal{A}}, \Pi, q \rightarrow p}{! \Gamma_{\mathcal{A}}, \Pi \rightarrow p / q}(\rightarrow /) \quad r \rightarrow r}{r /(p / q), ! \Gamma_{\mathcal{A}}, \Pi \rightarrow r} \\
& \frac{\frac{r(r /(p / q)), ! \Gamma_{\mathcal{A}}, \Pi \rightarrow r}{! \Gamma_{\mathcal{A}}, \Pi \rightarrow r}}{\left.\frac{!}{!} \rightarrow \rightarrow\right)}\left(\text { contr, } \text { perm }_{1}\right)^{*}
\end{aligned}
$$


Here, again, $\Pi$ is not empty and consists of non-bang-formulae, therefore we can legally apply $(! \rightarrow)$ and $(\rightarrow /)$.

$\Leftrightarrow$ For deriving sequents of the form $! \Gamma, \Pi \rightarrow A$, where $\Gamma, \Pi$, and $A$ do not contain the exponential, one can use a simpler calculus than $\mathbf{E L}^{-}$:

$$
! \Gamma, p \rightarrow p
$$

$\frac{! \Gamma, \Pi, B \rightarrow A}{! \Gamma, \Pi \rightarrow A / B}(\rightarrow /)$, where $\Pi \neq \Lambda \quad \frac{! \Gamma, \Pi \rightarrow B \quad ! \Gamma, \Delta_{1}, A, \Delta_{2} \rightarrow C}{! \Gamma, \Delta_{1}, A / B, \Pi, \Delta_{2} \rightarrow C}(/ \rightarrow)$

$\frac{! \Gamma, \Delta_{1}, B, \Delta_{2} \rightarrow A}{! \Gamma, \Delta_{1}, \Delta_{2} \rightarrow A}(! \rightarrow)$, where $B$ is a type from $\Gamma$ and $\Delta_{1}, \Delta_{2} \neq \Lambda$

Here (weak) is hidden into the axiom, (contr) comes within $(\rightarrow /)$, and $(! \rightarrow)$ includes both $\left(\right.$ perm $\left._{i}\right)$ and (contr) in the needed form. One can easily see that if $\mathbf{E L}^{-}, \vdash ! \Gamma, \Pi \rightarrow A$, where $\Gamma, \Pi$, and $A$ do not contain!, then this sequent is derivable in the simplified calculus. Moreover, the $(! \rightarrow)$ rule is interchangeable with the others in the following ways:

$$
\begin{gathered}
\frac{! \Gamma, \Delta_{1}, C, \Delta_{2}, B \rightarrow A}{! \Gamma, \Delta_{1}, C, \Delta_{2} \rightarrow A / B}(\rightarrow /) \quad \rightsquigarrow \quad \frac{! \Gamma, \Delta_{1}, C, \Delta_{2}, B \rightarrow A}{! \Gamma, \Delta_{1}, \Delta_{2} \rightarrow A / B}(! \rightarrow) \\
\frac{! \Gamma, \Delta_{2}, B \rightarrow A}{! \Gamma, \Delta_{1}, \Delta_{2} \rightarrow A / B}(\rightarrow /) \\
\frac{! \Gamma, \Pi \rightarrow B \quad ! \Gamma, \Delta_{1}, A, \Delta_{2}^{\prime}, D, \Delta_{2}^{\prime \prime} \rightarrow C}{\frac{! \Gamma, \Delta_{1}, A / B, \Pi, \Delta_{2}^{\prime}, D, \Delta_{2}^{\prime \prime} \rightarrow C}{! \Gamma, \Delta_{1}, A / B, \Pi, \Delta_{2}^{\prime}, \Delta_{2}^{\prime \prime} \rightarrow C}(! \rightarrow)} \\
\vdots \\
\frac{! \Gamma, \Delta_{1}, A, \Delta_{2}^{\prime}, D, \Delta_{2}^{\prime \prime} \rightarrow C}{! \Gamma, \Delta_{1}, A, \Delta_{2}^{\prime}, \Delta_{2}^{\prime \prime} \rightarrow C}(! \rightarrow) \\
\frac{! \Gamma, \Pi \rightarrow B}{! \Gamma, \Delta_{1}, A / B, \Pi, \Delta_{2}^{\prime}, \Delta_{2}^{\prime \prime} \rightarrow C}(/)
\end{gathered}
$$

And the same, if $D$ appears inside $\Delta_{1}$ or $\Pi$. Finally, consecutive applications of $(! \rightarrow)$ are always interchangeable.

After applying these transformations, we achieve a derivation where $(! \rightarrow)$ is applied immediately after applying $(/ \rightarrow)$ with the same active type (the other case, when it is applied after the axiom to $p$, is impossible, since then it 
violates the non-emptiness condition). In other words, applications of $(! \rightarrow)$ appear only in the following two situations:

$$
\frac{! \Gamma, \Pi \rightarrow p \quad ! \Gamma, \Delta_{1}, r / q, \Delta_{2} \rightarrow A}{\frac{! \Gamma, \Delta_{1},(r / q) / p, \Pi, \Delta_{2} \rightarrow A}{! \Gamma, \Delta_{1}, \Pi, \Delta_{2} \rightarrow A}(! \rightarrow)}(\rightarrow)
$$

and

$$
\frac{! \Gamma, \Pi \rightarrow p / q \quad ! \Gamma, \Delta_{1}, r, \Delta_{2} \rightarrow A}{\frac{! \Gamma, \Delta_{1}, r /(p / q), \Pi, \Delta_{2} \rightarrow A}{! \Gamma, \Delta_{1}, \Pi, \Delta_{2} \rightarrow A}(! \rightarrow)}(\rightarrow)
$$

Now we prove the statement $\mathbf{E L}^{-} \vdash ! \Gamma_{\mathcal{A}}, \Pi \rightarrow A \Rightarrow \mathbf{L}+\mathcal{A} \vdash \Pi \rightarrow A$ by induction on the above canonical derivation. If $! \Gamma_{\mathcal{A}}, \Pi \rightarrow A$ is an axiom or is obtained by an application of $(/ \rightarrow)$ or $(\rightarrow /)$, we apply the corresponding rules in $\mathbf{L}+\mathcal{A}$, so the only interesting case is $(! \rightarrow)$. Consider the two possible situations.

In the $(r / q) / p$ case, by induction hypothesis we get $\mathbf{L}+\mathcal{A} \vdash \Pi \rightarrow p$ and $\mathbf{L}+\mathcal{A} \vdash \Delta_{1}, r / q, \Delta_{2} \rightarrow A$, and then we develop the following derivation in $\mathbf{L}+\mathcal{A}$ (recall that (cut) is admissible there):

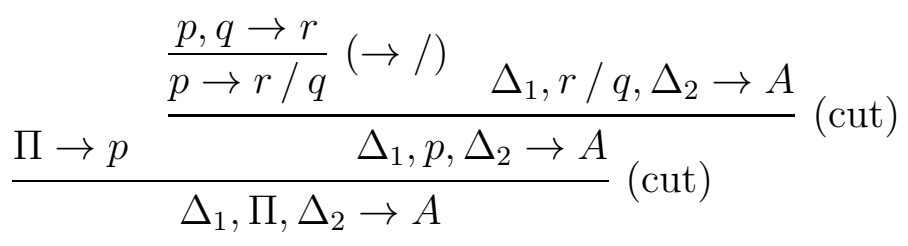

In the case of $r /(p / q)$, the derivation looks like this:

$$
\frac{\Pi \rightarrow p / q \quad \frac{p / q \rightarrow r \quad \Delta_{1}, r, \Delta_{2} \rightarrow A}{\Delta_{1}, p / q, \Delta_{2} \rightarrow A} \text { (cut) }}{\Delta_{1}, \Pi, \Delta_{2} \rightarrow A} \text { cut) }
$$

Note that in this proof we do not need any form of the cut rule for $\mathbf{E L}^{-}$. Theorem 12. The derivability problem for $\mathbf{E L} \mathbf{m}_{/}^{\mathrm{mk}}$ (and, thus, for $\mathbf{E L}^{\mathrm{mk}}$ ) is undecidable.

Proof. By Lemma 6.1 we have that if $\Gamma, \Pi$, and $A$ do not contain !, then $\mathbf{E L}_{/}^{\mathrm{mk}} \vdash ! \Gamma, \Pi \rightarrow A \Longleftrightarrow \mathbf{E L}^{-}, \vdash ! \Gamma, \Pi \rightarrow A$.

Of course, everything discussed above can be dually performed for \ instead of /, yielding undecidability for $\mathbf{E L}_{\backslash}^{\mathrm{mk}}$ and $\mathbf{E L}^{-} \backslash$. 


\section{Conclusion}

The derivability problem for the original Lambek calculus, without exponential modalities, is decidable and belongs to the NP class. This happens because the cut-free proof of a sequent has linear size with respect to the sequent's length. For the full Lambek calculus [21] and for its fragments with any two of three connectives (two divisions [22] or one division and the product [23]) the derivability problem is NP-complete.

On the other hand, for derivability problem in $\mathbf{L}$ / there exists a polynomial time algorithm [24]. Thus the one-division fragment of the Lambek calculus appears to be significantly simpler. Despite this, in our undecidability results for $\mathbf{E L}^{-}$and $\mathbf{E} \mathbf{L}^{\mathrm{mk}}$ we use only one of the two divisions.

\section{Related Work}

In the case of commutative linear logic Nigam and Miller [25] consider calculi that have several modalities interacting with each other, and different modalities are controlled by different sets of structural rules. These modalities are called subexponentials. A systematic study of subexponentials in the non-commutative case, under the umbrella of the Lambek calculus and cyclic linear logic, is performed in [26]. We also plan to study systems with (sub)exponentials, built on top of light [27] and soft [28] linear logic. Some preliminary results in this direction were presented at the 2018 Mal'tsev Meeting [29].

It appears that the technique used in the $\Leftarrow$ part of the proof of Lemma 9.1 is an non-commutative instance of focusing [30, 31]. Focusing techniques in the non-commutative case, for the Lambek calculus extended with subexponentials, are developed in [32].

\section{Acknowledgments}

This research was performed in part during visits of Stepan Kuznetsov and Max Kanovich to the University of Pennsylvania. We greatly appreciate support of the Mathematics Department of the University. A part of the work was also done during the stay of Andre Scedrov at the National Research University Higher School of Economics. We would like to thank S. O. Kuznetsov and I. A. Makarov for hosting us there.

This article was prepared within the framework of the HSE University Basic Research Program and funded by the Russian Academic Excellence Project '5-100.' 


\section{References}

\section{References}

[1] J. Lambek, The mathematics of sentence structure, American Mathematical Monthly 65 (1958) 154-170. doi:10.2307/2310058.

[2] B. Carpenter, Type-logical semantics, MIT Press, 1998.

[3] G. V. Morrill, Categorial grammar: logical syntax, semantics, and processing, Oxford University Press, 2011.

[4] R. Moot, C. Retoré, The logic of categorial grammars. A deductive account of natural language syntax and semantics, Vol. 6850 of Lecture Notes in Computer Science, Springer, 2012. doi:10.1007/978-3-642-31555-8.

[5] J. Lambek, On the calculus of syntactic types, in: R. Jakobson (Ed.), Structure of Language and Its Mathematical Aspects, Vol. 12 of Proceedings of Symposia in Applied Mathematics, AMS, Providence, RI, 1961, pp. 166-178.

[6] V. M. Abrusci, A comparison between Lambek syntactic calculus and intuitionistic linear logic, Zeitschrift für mathematische Logik und Grundlagen der Mathematik (Mathematical Logic Quarterly) 36 (1990) 11-15. doi:10.1002/malq.19900360103.

[7] D. N. Yetter, Quantales and (noncommutative) linear logic, Journal of Symbolic Logic 55 (1) (1990) 41-64. doi:10.2307/2274953.

[8] J.-Y. Girard, Linear logic, Theoretical Computer Science 50 (1) (1987) 1-102. doi:10.1016/0304-3975(87)90045-4.

[9] M. Kanovich, The expressive power of modalized purely implicational calculi, CSLI Report, Stanford University (1993).

[10] P. de Groote, On the expressive power of the Lambek calculus extended with a structural modality, in: C. Casadio, P. J. Scott, R. A. G. Seely (Eds.), Language and Grammar. Studies in Mathematical Linguistics and Natural Language, Vol. 168 of CSLI Lecture Notes, 2005, pp. 95111. 
[11] M. Kanazawa, Lambek calculus: Recognizing power and complexity, in: J. D. Gerbrandy, M. J. Marx, M. de Rijke, Y. Venema (Eds.), JFAK. Essays dedicated to Johan van Benthem to the occasion of his 50th birthday, Vossiuspers, Amsterdam University Press, 1999.

[12] P. Lincoln, J. Mitchell, A. Scedrov, N. Shankar, Decision problems for propositional linear logic, Annals of Pure and Applied Logic 56 (1) (1992) 239-311. doi:10.1016/0168-0072(92)90075-B.

[13] M. Kanovich, The undecidability theorem for the Horn-like fragment of linear logic (Revisited), Mathematical Structures in Computer Science 26 (5) (2016) 719-744. doi:10.1017/S0960129516000049.

[14] M. I. Kanovich, Simulating linear logic in 1-only linear logic, CNRS, Laboratoire de Mathématiques Discrétes. Prétirage nº 94-02 (1994).

[15] M. I. Kanovich, The complexity of neutrals in linear logic, in: Proceedings of Tenth Annual IEEE Symposium on Logic in Computer Science (LICS 1995), IEEE, 1995, pp. 486-495. doi:10.1109/LICS.1995.523282.

[16] H. Hendriks, Studied flexibility, Ph.D. thesis, Institute for Logic, Language and Information, Amsterdam, ILLC Dissertation Series, DS-199305 (1993).

[17] F. Métayer, Polynomial equivalence among systems LLNC, $\mathrm{LLNC}_{a}$ and $\mathrm{LLNC}_{0}$, Theoretical Computer Science 227 (1-2) (1999) 221-229. doi:10.1016/S0304-3975(99)00053-5.

[18] S. Kuznetsov, Lambek grammars with one division and one primitive type, Logic Journal of the IGPL 20 (1) (2012) 207-221. doi:10.1093/jigpal/jzr031.

[19] W. Buszkowski, Some decision problems in the theory of syntactic categories, Zeitschrift für mathematische Logik und Grundlagen der Mathematik (Mathematical Logic Quarterly) 28 (1982) 539-548. doi:10.1002/malq.19820283308.

[20] M. Pentus, Lambek grammars are context-free, in: Proceedings of Eighth Annual IEEE Symposium on Logic in Computer Science (LICS 1993), IEEE, 1993, pp. 429-433. doi:10.1109/LICS.1993.287565. 
[21] M. Pentus, Lambek calculus is NP-complete, Theoretical Computer Science 357 (1) (2006) 186-201. doi:10.1016/j.tcs.2006.03.018.

[22] Y. Savateev, Product-free Lambek calculus is NP-complete, in: S. Artemov, A. Nerode (Eds.), Logical Foundations of Computer Science (LFCS 2009), Vol. 5407 of Lecture Notes in Computer Science, Springer, 2009, pp. 380-394. doi:10.1007/978-3-540-92687-0_26.

[23] Y. Savateev, Algorithmic complexity of fragments of the Lambek calculus, Ph.D. thesis, Moscow State University, (in Russian) (2009).

[24] Y. Savateev, Lambek grammars with one division are decidable in polynomial time, in: E. A. Hirsch, A. A. Razborov, A. Semenov, A. Slissenko (Eds.), Computer Science - Theory and Applications (CSR 2008), Vol. 5010 of Lecture Notes in Computer Science, Springer, 2008, pp. 273-282. doi:10.1007/978-3-540-79709-8_28.

[25] V. Nigam, D. Miller, Algorithmic specifications in linear logic with subexponentials, in: Proceedings of the 11th ACM SIGPLAN Conference on Principles and Practice of Declarative Programming (PPDP '09), ACM, 2009, pp. 129-140. doi:10.1145/1599410.1599427.

[26] M. Kanovich, S. Kuznetsov, V. Nigam, A. Scedrov, Subexponentials in non-commutative linear logic, Mathematical Structures in Computer ScienceFirstView (published online). doi:10.1017/S0960129518000117.

[27] J.-Y. Girard, Light linear logic, Information and Computation 143 (2) (1998) 175-204. doi:10.1006/inco.1998.2700.

[28] Y. Lafont, Soft linear logic and polynomial time, Theoretical Computer Science 318 (1-2) (2004) 163-180. doi:10.1016/j.tcs.2003.10.018

[29] M. Kanovich, S. Kuznetsov, A. Scedrov, Lambek calculus enriched with multiplexing, in: Mal'tsev Meeting. Collection of Abstracts, Sobolev Institute of Mathematics and Novosibirsk State University, Novosibirsk, 2018.

[30] J.-M. Andreoli, Logic programming with focusing proofs in linear logic, Journal of Logic and Computation 2 (3) (1992) 297-347. doi:10.1093/logcom/2.3.297. 
[31] V. Nigam, D. Miller, Focusing in linear meta-logic, in: A. Armando, P. Baumgartner, G. Dowek (Eds.), Automated Reasoning (IJCAR 2008), Vol. 5195 of Lecture Notes in Artificial Intelligence, Springer, 2008, pp. 507-522. doi:10.1007/978-3-540-71070-7_42.

[32] M. Kanovich, S. Kuznetsov, V. Nigam, A. Scedrov, A logical framework with commutative and non-commutative subexponentials, in: D. Galmiche, S. Schulz, R. Sebastiani (Eds.), Automated Reasoning (IJCAR 2018), Vol. 10900 of Lecture Notes in Artificial Intelligence, Springer, 2018, pp. 228-245. doi:10.1007/978-3-319-94205-6_16. 